\title{
The perception of offset: A problem of decision criteria
}

\author{
M. J. PENNER \\ Hunter College of the City University of New York, 695 Park Avenue, New York, New York 10021
}

\begin{abstract}
Robinson (1974) has presented a simple form of a hypothesis which assumes that the sensory response to a stimulus persists for a period of time exceeding its physical duration. He uses this hypothesis to predict Efron's (1970) results concerning the delayed perception of offset of brief stimuli. We propose a natural extension of this hypothesis that assumes that each subject adopts a "personal" criterion in order to judge offset. This model not only provides predictions consistent with Efron's data, but can also predict data of quite dissimilar appearance, including the data of the present study.
\end{abstract}

\section{INTRODUCTION AND THEORY}

It has long been assumed that the perception of a sensory stimulus outlasts its physical presence. Much data supports this view, at least in rough form (e.g., see Sperling, 1960, for work in vision; Plomp, 1964, for work in audition). Another well-established hypothesis holds that perception of brief stimuli results from the integration or averaging of sensory stimulation over a period of time. The evidence favoring this view comes from studies showing a reciprocal relation of duration and intensity for threshold detection of brief stimuli in either vision (Block's law) or audition (Garner \& Miller, 1947).

The two hypotheses of persistence and integration have often served as a base for models of sensory phenomena. In the present case, we wish to discuss how these hypotheses can be used to predict data concerning the perception of offset of brief stimuli. Robinson (1974) has mathematized these notions by proposing a "running-average" model of this type:

$$
y(t)=\int_{0}^{\infty} \omega(\tau) x(t-\tau) d \tau
$$

Here $x(t)$ represents the sensory input (e.g., intensity as a function of time), $y(t)$ represents the perception upon which the subject bases his response, and $\omega(\tau)$ is an averaging mechanism that integrates sensory stimulation, thereby causing sensory persistence. In other words, if $\omega(\tau)$ exceeds zero after the stimulus has physically stopped, the perception will persist for longer than the stimulus. The shape of $\omega(\tau)$ is an important factor which, for the sake of simplicity, Robinson assumed to be rectangular, so that

$$
\begin{aligned}
\omega(\mathrm{t}) & =\mathrm{K} & & 0 \leqslant \mathrm{t} \leqslant \mathrm{T} \\
& =0 & & \text { otherwise }
\end{aligned}
$$

This research was supported in part by Grant $10772 \mathrm{~N}$ from the City University of New York and in part by NHI. HEW, Public Health Service Grant 1R01NS12085-01.

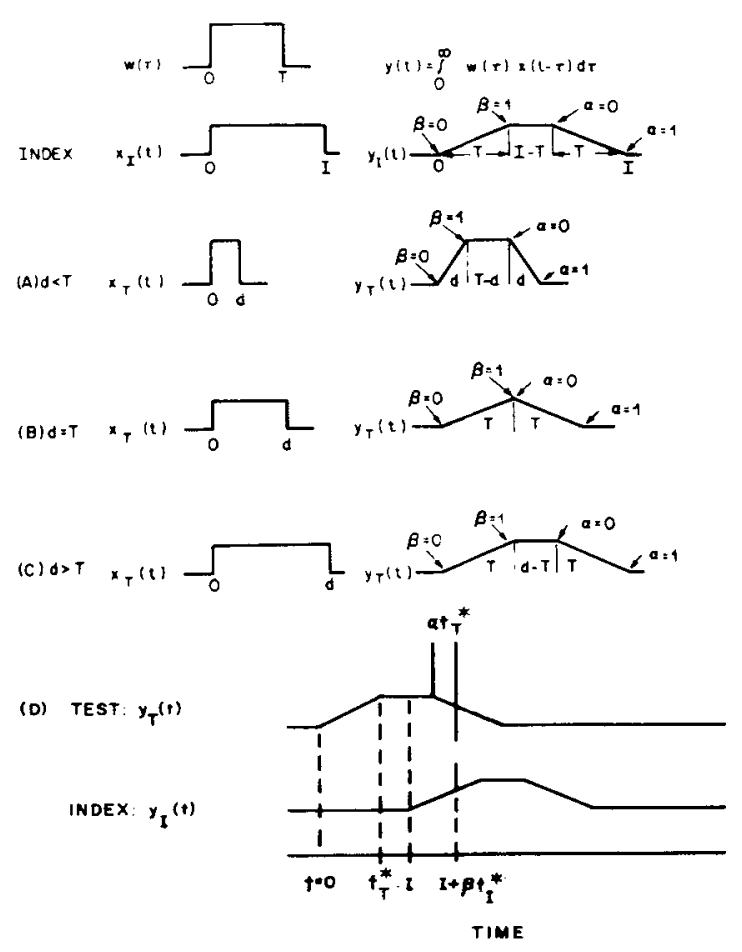

Figure 1. Schematic representation of the model. The weighting function is $\omega(\tau)$ and lasts $T$ msec. $T$ was termed the critical duration by Efron. The output, $y(t)$, is the convolution of $\omega(\tau)$ with the input waveform, $x(t)$. In Panel $A, y_{T}(t)$ is drawn for an $x(t)$ of duration $d$, where $d<T$. In Panel $B, y_{T}(t)$ is drawn for an $x(t)$ of duration $d$, where $d=T$. In Panel $C, y_{T}(t)$ is drawn for $x(t)$ of duration $d$, where $d>T$. In Panel $D$, a test stimulus and an index are both drawn and represent a possible lineup for an offet decision.

Hence

$$
y(t)=K \int_{0}^{T} x(t-\tau) d \tau
$$

In Figure 1, $y(t)$ is shown for various durations of $x(t)$ under the assumption that $\omega(\tau)$ is rectangular and that $x(t)$ is rectangularly gated on and off. Note that each $y(t)$ rises and falls linearly. The rise and fall times are always equal and will be denoted by $t^{*}$. When the stimulus duration, $d$, is less than $T$ [the 
integration time of $\omega(\tau)]$, then $t^{*}=d$; when $d \geqslant T$, then $\mathrm{t}^{*}=\mathrm{T}$.

Let us now consider how subjects might judge the oftset (and onset) of stimuli if their perceptions [ $y(t)]$ are described by Equation 3. A "perfect" decision maker would judge onset of a stimulus to occur when $y(t)$ begins to rise, which happens at about the moment of physical onset, and would judge offset to occur when $y(t)$ begins to fall. Indeed. this was the decision assumption adopted by Robinson (1974). However, a more realistic assumption is that the subject judges onset to have occurred when the rising part of $y(t)$ exceeds some criterion and offset to have occurred when the falling part of $y(t)$ drops below some criterion. In particular, let us make the reasonable assumption that the subject judges onset to have occurred at time $\beta t^{*}$ after the start of the rising limb, and judges offset to have occurred at time $a^{*}$ after the start of the falling limb. These assumptions concerning the criteria $a$ and $\beta$ are chosen because a constancy in their values across various conditions reflects a constant decision rule for detection of a change in slope: namely, the slope change is detected when a decibel shift larger than some constant value has occurred.

We shall see below that this simple extension of the Robinson model leads to predictions that depend on the choice of the criteria, $a$ and $\beta$. When $a$ and $\beta$ are zero, then the model reduces to that of Robinson (1974) and makes predictions in accord with Efron's (1970) data. Of course, only a perfect subject could adopt zero values for $a$ and $\beta$ in the context of this model. since zero values imply that the subject perceives a change in the slope of $y(t)$ in zero time.

\section{EMPIRICAL RESULTS}

We now consider an experiment attempting to measure perceived offset of a simple stimulus. Efron (1970) presented subjects with a test stimulus to one ear (a noise burst of variable duration) and an index to the other ear (a $500-\mathrm{msec}, 2,000-\mathrm{Hz}$ tone). The subject adjusted these stimuli so that the onset of the index marked the offset of the test stimulus. The interval, $D$, between the offset of the test stimulus and the onset of the index was assumed to measure the error in perceiving the offset of the test stimulus. Rather surprisingly, for brief test stimuli, the perception of the test stimulus's offset occurred at a fixed interval of time after the test stimulus's onset.

Efron's results can be summarized in a graph relating $\mathrm{D}$ to the test stimulus's duration, $d$. These data are very well titted by the function

$$
\begin{array}{rlrl}
\mathbf{D} & =\mathrm{T}-\mathrm{d} & \mathrm{d} \leqslant \mathrm{T} \\
& =\mathrm{K} & \mathrm{d}>\mathrm{T},
\end{array}
$$
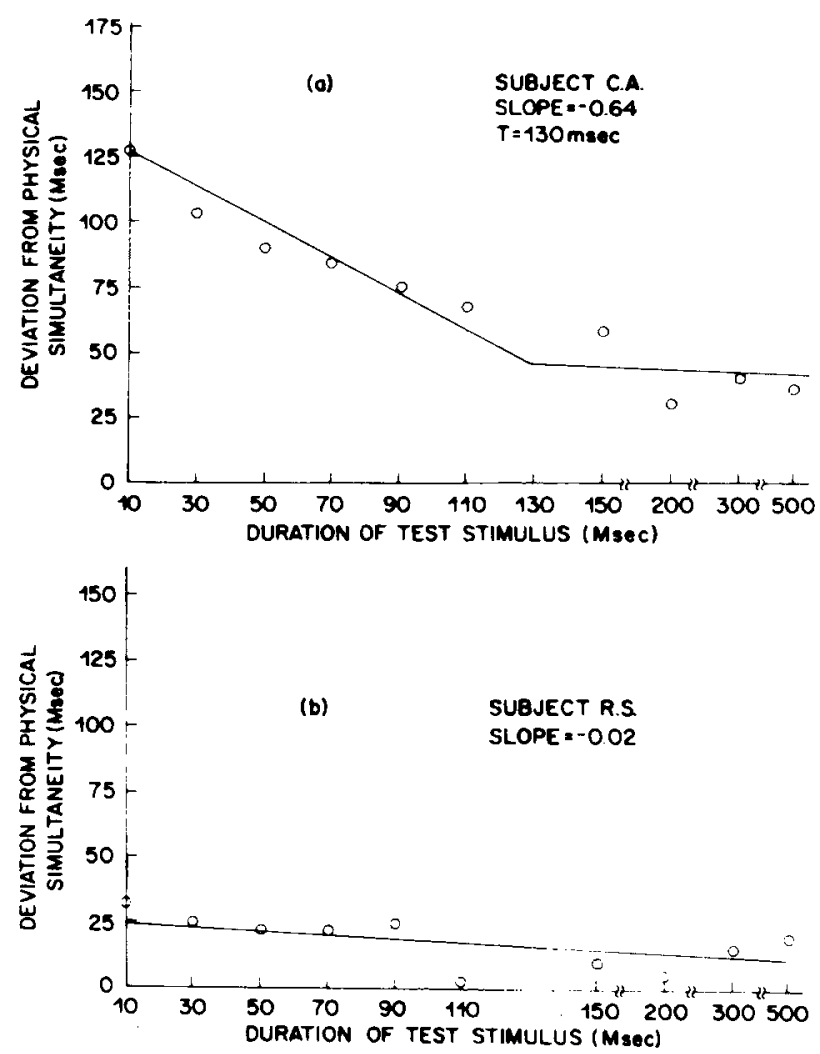

Figure 2. Graph of the deviation from physical simultaneity, D, as a function of the test stimulus's duration. The circles represent the data from the first experiment and the Xs represent the data from the second experiment. The top graph represents C.A.'s data, and the bottom graph represents R.S.'s data.

where $T$ is about $130 \mathrm{msec}$ and is called the critical duration. Note that the slope of the line relating $D$ to $\mathrm{d}$ is -1.0 .

It is, however, possible to replicate Efron's experiment and yet to obtain data which do not replicate his findings. Figure 2 displays the results of such a replication for two subjects. The procedure is the same as in Efron's (1970) study, although the data are not consistent with his. Furthermore, the two subjects produce data that are quite dissimilar. Intersubject variability on such a subjective task is not surprising but, nonetheless, merits some explanation. $^{1}$

\section{DERIVATIONS AND APPLICATIONS OF THE MODEL}

One explanation of these data and Efron's can be simply obtained by applying the model summarized by Equation 3 to the data. Suppose that $a=0$ and $\beta=0$ (Robinson's model); then offset is perceived when the physical start of the index stimulus is placed by the subject at the start of the descending limb of $y(t)$ for the test stimulus. Reference to Figure 1 makes 
it clear that when $d \leqslant t, D$ (the physical offset-onset difference) will be equal to $\mathrm{T}-\mathrm{d}$. For $\mathrm{d}>\mathrm{T}, \mathrm{D}$ will be equal to zero. Thus, Robinson's model predicts the form of Efron's data. The form of Efron's data suggests that the parameter $a$ is quite small for the subjects in his experiment.

On the other hand, suppose that $a$ is not small: suppose that the subject cannot perceive a change in the shape of $y(t)$ in nearly zero time. The schematic in Figure $1 \mathrm{D}$ helps to understand the results in this case. The index stimulus's onset occurs at time I. The difference in time between the physical onset of the inde $x$ and the physical offset of the test is a measure of the error. D, in judging offset. Thus,

$$
\mathrm{D}=\mathrm{I}-\mathrm{d}
$$

Recall that the rise and decay time of any stimulus is defined as $t^{*}$. When the stimulus is of duration $d$ where $\mathrm{d} \leqslant \mathrm{T}$, then $\mathrm{t}^{*}=\mathrm{d}$; when $\mathrm{d}>\mathrm{T}$, then $\mathrm{t}^{*}=\mathrm{T}$. With the help of Figure 1, we now simply replace I in Equation 4 by its value in terms of $d, T, a$, and $\beta$. When $d \leqslant T$, the descending limb of $y(t)$ for the test stimulus begins at time $\mathrm{T}$ (see Figure $1 \mathrm{~A}$ ). Thus $\mathrm{I}=\mathrm{T}+a \mathrm{t}_{\mathrm{T}}^{*}-\beta \mathrm{t}_{\mathrm{I}}^{*}$ (see Figure 1D). Hence, substituting in Equation 4,

$$
\mathrm{D}=\left(\mathrm{T}+a \mathrm{t}_{\mathrm{T}}^{*}-\beta \mathrm{t}_{\mathrm{I}}^{*}\right)-(\mathrm{d}) \quad \mathrm{d} \leqslant \mathrm{T} .
$$

Now $\mathrm{t}_{\mathrm{T}}^{*}=\mathrm{d}$ since $\mathrm{d} \leqslant \mathrm{T}$ and $\mathrm{t}_{\mathrm{I}}^{*}=\mathrm{T}$ since the index (500 $\mathrm{msec}$ ) is certainly longer than $T$ and thus, substituting in Equation 5 ,

$$
\begin{aligned}
\mathrm{D} & =\mathrm{T}+a \mathrm{~d}-\beta \mathrm{T}-\mathrm{d} \quad \mathrm{d} \leqslant \mathrm{T} \\
& =(1-\beta) \mathrm{T}+(a-1) \mathrm{d} .
\end{aligned}
$$

When $d>T$, the descending limb of $y(t)$ for the test stimulus begins at time $d$ (see Figure $1 \mathrm{C}$ ). Thus $\mathrm{I}=\mathrm{d}+$ $a \mathrm{t}_{\mathrm{T}}^{*}-\beta \mathrm{t}_{\mathrm{I}}^{*}$ (see Figure 1D). Hence, substituting in Equation 4,

$$
\mathrm{D}=\left(\mathrm{d}+a \mathrm{t}_{\mathrm{T}}^{*}-\beta \mathrm{t}_{\mathrm{I}}^{*}\right)-(\mathrm{d}) \quad \mathrm{d}>\mathrm{T}
$$

Now, $\mathrm{t}_{\mathrm{T}}^{*}=\mathrm{T}$ since $\mathrm{d}>\mathrm{T}$ and $\mathrm{t}_{\mathrm{T}}^{*}=\mathrm{T}$ since the index is again longer than $T$, so that substituting in Equation 7 ,

$$
\mathrm{D}=\mathrm{d}+a \mathrm{~T}-\beta \mathrm{T}-\mathrm{d}=(a-\beta) \mathrm{T} \quad \mathrm{d}>\mathrm{T}
$$

Equations 6 and 8 specify the general form of the function relating $\mathrm{D}$ to $\mathrm{d}$ for index duration $>\mathrm{T}$. The predictions are graphed in Figure 3A. From Equation 6, for $d \leqslant T$, the function is linear with a slope determined by a. From Equation 8, for $\mathrm{d}>\mathrm{T}$, the function is linear with zero slope. According to the model, then, the function relating $D$ to $d$ will have a descending limb with a slope between 0 and -1.0
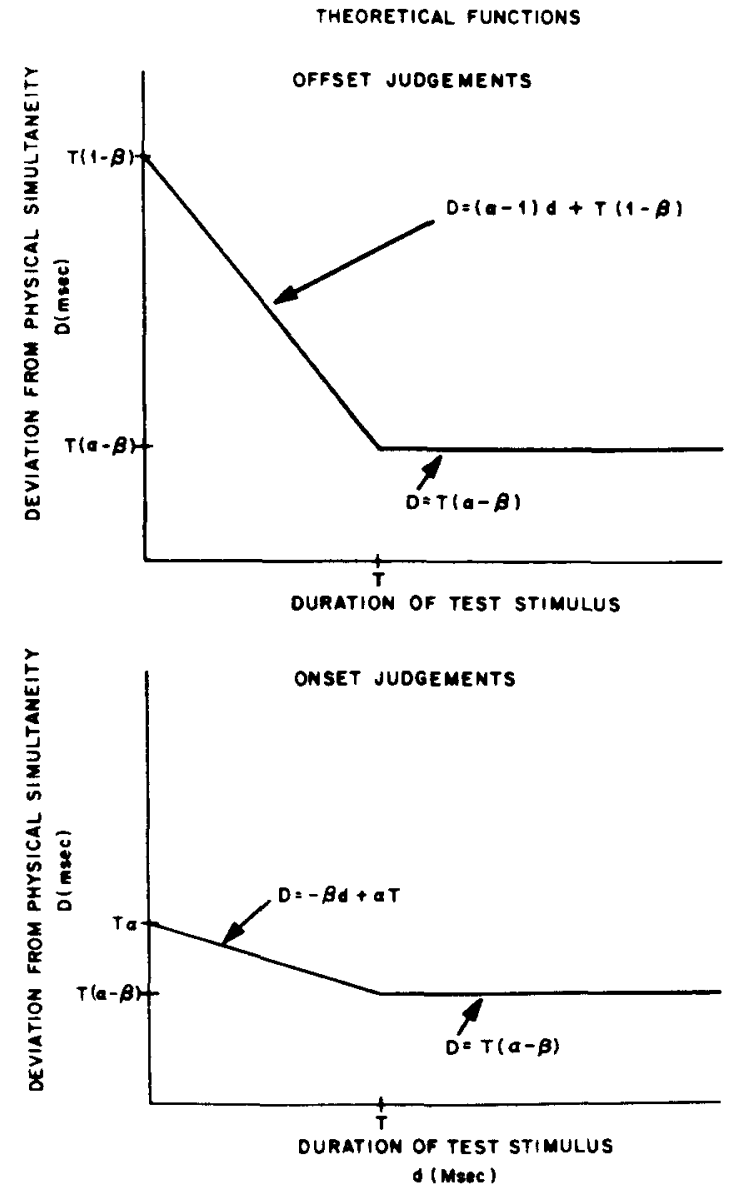

Figure 3. Theoretical predictions of the model. On the ordinate is the deviation from physical simultaneity, D; on the abscissa is the test stimulus's duration, $d$. The general form of $D$ for offset judgments is shown in Panel $A$. The general form for onset judgments is shown in Panel B.

followed by a limb with zero slope (i.e., constant error). Thus, depending on the values of the criteria, $a$ and $\beta$, the model predicts either Efron's data or the data presented in Figure 2. In Figure 2, the solid lines represent the predictions of the model for values of $a$ and $\beta$ chosen to minimize the sum of the least square deviations of the predictions from the data. For Subject C.S., $a=.36$ and $\beta=0.11$; for Subject R.S., $a=0.98$ and no estimate of $\beta$ was possible.

By reversing the index and the test stimuli in Figure 1D, we may develop predictions from this model for a similar experiment measuring the perception of onset. The predictions are shown in Figure $3 B$. If $\beta$ is estimated to be very small, as in the offset predictions, an essentially flat line is predicted. This was found both in Efron's data and in a replication we carried out. In our case, we found constant errors of about 43 and $13 \mathrm{msec}$ for Subjects C.A. and R.S.

At this point, one should ask how the predictions would change it a different integrator shape was 
posited. For example, a fast exponential rise and slow exponential decay is a typical assumption in the literature (Duifhuis, 1973; Munson, 1947; Zwislocki, 1969). Although the calculations have not been carried out in detail, it seems safe to say that the choice of criteria will again have marked effects on offset judgments.

Finally, it should be noted that the details of the model are easily testable in a variety of ways. For example, the subject's criterion could be manipulated by verbal instructions, payoffs, or simply altered by the presence of a continuous background noise. Such tests are obviously desirable and will be undertaken. Nonetheless, the basic notion of criterion variability is usual in models of auditory phenomena: sensory judgments have traditionally been viewed as possessing criterional bases. Thus, although we have failed to replicate Efron, we have shown that a plausible criterion-based model similar in substance to Efron's can predict either his results or ours.

\section{CONCLUSION}

We have modified Robinson's (1974) model by assuming that each subject selects "personal" criteria for perceiving a stimulus's onset and offset. In this light. Efron's subjects can be seen to have selected one criterion for offset, whereas the subjects whose data are presented in Figure 2 can be seen to have selected another criterion. Thus, we have shown that data may be obtained which do not replicate Efron's, and that even data which do not replicate Efron's may be viewed as being consistent with the integration model incorporating a critical duration, $T$.

\section{REFERENCES}

Duifhuss, H. Consequences of peripheral frequency analysis for nonsimultaneous masking. Journal of the Acoustical Society of America, 1973, 54, 1471-1488.
Erron, R. The relationship between the duration of a stimulus and the duration of a perception. Neuropsychologia. 1970. 8. 37-55.

Garner. W. R., \& Miller, G. A. The masked threshold of pure tones as a function of duration. Joumal of Experimental Psychology. 1947. 37, 294-303.

Munson. W. A. The growth of auditory sensation. Journal of the Acoustical Society of America, 1947, 19. 584-591.

Plomp, R. Rate of decay of auditory sensation. Journal of the Acoustical Society of America, 1964, 36. 277-282.

Robinson. C. E. Reaction time to the offset of brief auditory stimuli. Perception \& Psychophysics, 1973. 13. 281-283.

Robinson. C. E. Simple form of the auditory running-average hypothesis: Application to the temporal summation of loudness and to the delayed perception of offset of brief stimuli. Journal of the Acoustical Society of A merica, 1974, 55, 645-649.

SPERLING, G. The information available in brief visual presentations. Psychological Monographs, 1960, 74(11, Whole No. 498).

Zwislocki, J. J. Temporal summation of loudness: An analysis. Journal of the Acoustical Society of America, 1969, 46. 431-441.

\section{NOTE}

1. The offset judgments were rerun on three additional subjects. The test stimuli were 10 and $100 \mathrm{msec}$, and the slopes of the lines relating $D$ to $d$ were $-0.71,+0.28$, and -0.04 . These results confirm the variability of slopes seen in this experiment. Another lack of correspondence with Efron's research was noted by Robinson (1973), who measured the reaction time to offset of stimuli ranging from 5 to $200 \mathrm{msec}$. He reasoned that if the perception of offset occurred at a fixed interval of time after the stimulus's onset, then reaction time to offset would also occur at a fixed interval of time after the test stimulus's onset. He found. however, that the reaction time to the offset of a stimulus was about $185 \mathrm{msec}$, independent of its duration. We can account for both results with the criterion-based model presented in the next section if $T$ is very brief or if $\alpha$ is one. This value of $\alpha$ is surprising, since it implies that the subject waits until decay is complete to respond to the stimulus offset.
(Received for publication May 24, 1974; revision received January 13,1975 .) 